\title{
Identificación de los polimorfismos G1 y G8 del gen GDF9 en ovinos criollos Araucanos ${ }^{\#}$
}

\author{
Identification of G1 and G8 polymorphisms of GDF9 gene in Araucano creole sheep
}

\author{
E Paz ${ }^{\mathrm{a}, \mathrm{b}}, \mathrm{J}$ Quiñones ${ }^{\mathrm{a}, \mathrm{b}}, \mathrm{S}$ Bravo $^{\mathrm{b}}, \mathrm{E}_{\text {Rodero }}^{\mathrm{c}}$, A Gonzalez ${ }^{\mathrm{c}}$, N Sepúlveda ${ }^{\mathrm{b} *}$

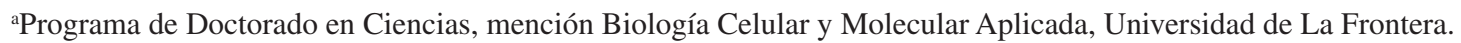 \\ bLaboratorio de Producción Animal, Facultad de Ciencias Agropecuarias y Forestales, Universidad de La Frontera, Temuco, Chile. \\ 'Departamento de Producción Animal, Universidad de Córdoba, España.
}

\begin{abstract}
SUMMARY
Polymorphisms in major gene effect related to reproductive activity have been described in sheep. Mutations located in the GDF9 gene have been associated with increased ovulation rate and litter size. GDF9 factor is secreted by the oocyte and is a member of the family of transforming growth factors (TGF- $\beta$ ) located on chromosome 5. The aim of this study was to identify the presence of polymorphic regions (G1 and G8) in the GDF9 gene in Araucano creole sheep. DNA was extracted from 100 blood samples and the presence of mutations was determinated using PCR-RFLP. Two regions were amplified, one corresponding to exon 1 of $462 \mathrm{bp}$ digested with restriction enzyme HhaI and a 139bp fragment corresponding to exon 2 digested with enzyme DdeI. The presence of polymorphisms in G1 of GDF9 gene were detected with a genotype frequency of 0.56 (genotype GG), 0.44 (genotype GA) and an allele frequency of 0.78 for the allele (G) and 0.22 for the allele (A). No polymorphisms were detected in G8. This is the first report of this polymorphism in sheep in Chile that could serve as a genetic marker for marker for prolificacy selection in the the Araucano creole sheep.
\end{abstract}

Key words: prolificacy, sheep reproduction, molecular markers.

\section{RESUMEN}

En la especie ovina se han descrito una serie de polimorfismos en genes de efecto mayor relacionados con la actividad reproductiva. Las mutaciones ubicadas en el gen de efecto mayor GDF9 se han asociado con el incremento de la tasa ovulatoria y el tamaño de la camada. GDF9 es un factor celular secretado por el ovocito y es miembro de la familia de factores de crecimiento transformante (TGF- $\beta$ ) localizado en el cromosoma 5 ovino. El objetivo de este trabajo fue identificar la presencia de los polimorfismos en los sitios G1 y G8 en el gen GDF9 en ovinos criollos Araucanos. Se extrajo el ADN de 100 muestras sanguíneas para posteriormente determinar la presencia de las mutaciones utilizando la técnica PCR-RFLP. Fue amplificada una región de 462 pb correspondiente al exón 1, la cuál fue digerida con la enzima de restricción HhaI, el siguiente fragmento amplificado fue de 139 pb correspondiente al exón 2, siendo digerido con la enzima DdeI. Se identificó la presencia del polimorfismo G1 con una frecuencia genotípica del 0,56 (genotipo GG), 0,44 (genotipo GA) y una frecuencia alélica de 0,78 para el alelo (G) y 0,22 para el alelo (A). No se detectó la presencia de polimorfismo G8. Este es el primer reporte de este polimorfismo en ovinos en Chile que podría servir como un marcador genético de prolificidad para la selección de ovinos criollos Araucanos.

Palabras clave: prolificidad, reproducción ovina, marcadores moleculares.

\section{INTRODUCCIÓN}

Recientes progresos en genética molecular han hecho posible la búsqueda de genes candidatos que controlan rasgos reproductivos. La selección asistida por marcadores (MAS) utiliza secuencias de ADN que están asociadas con un rasgo específico, lo cual permite complementar los datos fenotípicos utilizados para la selección, basándose en una evaluación molecular directa (Mahmoud y Nawito 2012). Comúnmente se utilizan polimorfismos de nucleótido simple (SNP) para el desarrollo de marcado-

Aceptado: 03.10.2013.

\# Proyecto FONDECYT No 1120474 y DIUFRO DI12-101

* Av. Fco. Salazar 01145, P.O.Box 54-D, Temuco, Chile; nestor.sepulveda @ufrontera.cl res genéticos ya que proporcionan información puntual acerca de la variación alélica en un locus. La reacción en cadena de la polimerasa (PCR) asociada con la técnica de polimorfismos de longitud de fragmentos de restricción (RFLP) permite de manera rápida, simple y exacta genotipificar SNPs. Esta técnica requiere de enzimas de restricción que reconocen secuencias nucleotídicas específicas, permitiendo discriminar las diferentes variaciones alélicas de interés.

En varias razas ovinas se han descrito diferentes genotipos con efectos sobre la tasa ovulatoria y la prolificidad. La mayor parte de ellos están asociados a mutaciones localizadas en genes de efecto mayor BMPR1B, BMP15 y GDF9 pertenecientes a la superfamilia de los Transforming Growth Factor- $\beta$ (TGF- $\beta$ ). Muchos de estos factores de crecimiento son expresados por células somáticas 
del ovario y por ovocitos en crecimiento, con funciones reguladoras intraovaricas de la foliculogénesis (Knight y Glister 2006). En el estado preantral, GDF9 ha mostrado ser efectivo estimulando el crecimiento de folículos en ratas in vitro y promueve la sobrevida folicular suprimiendo la atresia y la apoptosis de las células de la granulosa (Otsuka y col 2011). También se ha reportado que las células del cúmulo requieren GDF9 previo al aumento de LH, para soportar la cascada metabólica, tales como glicólisis y biosíntesis de esteroles (Dekkers 2004). El locus GDF9 inhibe la esteroidogénesis inducida por FSH y la expresión del receptor de LH. Aumenta la regulación de la prostaglandina endoperóxido sintasa-2 (COX-2) y del receptor tipo 2 de las prostaglandinas, principalmente en células del cúmulo de folículos preovulatorios en ratas (Elvin y col 1999, Varani y col 2002). Diversos estudios han propuesto al locus GDF9 como marcador genético relacionado con la prolificidad. Hanrahan y col (2004) detectó 8 diferentes SNPs en ovinos, denominados G1, G2, G3, G4, G5, G6, G7 y G8. Investigaciones más recientes proponen 5 nuevos SNPs; FecG ${ }^{\text {SI }}$, FecTT, A152G, T692C y G729T en distintas razas (Chu y col 2011). Asociándose un aumento en la tasa ovulatoria con las mutaciones G1, G8, FecG ${ }^{\mathrm{SI}}$, FecTT, A152G, T692C y G729T principalmente en animales heterocigotos, mientras que animales homocigotos presentan bajas tasas ovulatorias (Chu y col 2007). Mediciones realizadas en ovejas, sugiere que el efecto de la mutación GDF-9 en la tasa ovulatoria es más grande que el de la mutación BMP-15, con una copia del alelo $\mathrm{FecG}^{\mathrm{H}}(\mathrm{G} 8)$, incrementando la tasa ovulatoria sobre 1,4 ovulaciones en las razas Cambridge y Belclare (Davis 2004). Sin embargo, la mayor parte de los análisis se concentran en el locus $\mathrm{G} 8\left(\mathrm{FecG}^{\mathrm{H}}\right)$ y G1 debido a que producen un aumento en el tamaño de camada (Hanrahan y col 2004, Moradband y col 2011). En la región de La Araucanía, Chile, existe un ovino criollo denominado Araucano, utilizado principalmente como animal de doble propósito por pequeños y medianos agricultores. Recientemente esta población fue caracterizada en sus aspectos morfológicos y productivos sugiriendo una aptitud más acorde a la producción de carne (Bravo y Sepúlveda 2010). Otros estudios han demostrado que posee altos índices reproductivos (Sepúlveda 1999, Sepúlveda y col 2001), pero se desconoce la existencia de algún factor genético relacionado a este rasgo. El objetivo de este trabajo fue identificar la presencia de los polimorfismos G1 y G8 del gen GDF9 en el ovino criollo Araucano, a través de la técnica PCR-RFLP.

\section{MATERIAL Y MÉTODOS}

Se extrajeron 100 muestras sanguíneas de ovejas adultas (2-4 partos) y machos con una edad promedio de 3,5 años. Estos animales pertenecen al Núcleo Genético de ovinos criollos Araucanos (NOA) de la Estación Experimental Maquehue de la Universidad de La Frontera, ubi- cada en la comuna de Freire, Región de La Araucanía, Chile. El NOA se formó hace 10 años, con ovinos provenientes de distintas comunas de la región (Padre Las Casas, Temuco, Vilcún, Lautaro, Cunco, Freire, Villarrica, Victoria, Melipeuco, Chol Chol y Nueva Imperial). Actualmente posee un total de 120 animales, los cuales se han seleccionado en los últimos 5 años por sus ganancias de peso vivo y prolificidad. Las hembras muestreadas se encontraban en fase de anestro estacional. La extracción de ADN genómico se realizó mediante el kit comercial Axyprep Blood Genomic DNA Miniprep (AxigenSci. Inc., CA, EUA). Fueron sintetizados los sets de partidores para amplificar dos regiones del gen GDF9 según lo descrito por Hanrahan y col (2004), un fragmento de 462 pb del exón 1 y 139 pb del exón 2 (cuadro 1). La PCR se realizó en un volumen final de $50 \mu \mathrm{l}$, conteniendo 100 ng de ADN genómico, 2,5 U Paq5000 polimerasa, 5 $\mu \mathrm{l}$ del buffer 10X Paq 5000 ${ }^{\mathrm{TM}}$, $500 \mu \mathrm{M}$ dNTPs, $250 \mathrm{nM}$ de los partidores forward y reverse. Las condiciones de amplificación para ambos fragmentos incluyeron una denaturación inicial de $95^{\circ} \mathrm{C}$ por 5 minutos, seguidos de 30 ciclos de denaturación a $95^{\circ} \mathrm{C}$ por 20 segundos, hibridación a $60^{\circ} \mathrm{C}$ por 20 segundos, extensión a $72^{\circ} \mathrm{C}$ por 30 segundos, y una extensión final de $72^{\circ} \mathrm{C}$ por 5 minutos. Posteriormente los productos fueron separados mediante una electroforesis en gel de agarosa al 1,5\% (p/v). Los productos de PCR fueron digeridos con las enzimas HhaI y Ddel respectivamente, en un volumen de reacción final de $25 \mu \mathrm{l}$. El mix de digestión se incubó por 7 horas a $37^{\circ} \mathrm{C}$, posteriormente los productos de la digestión fueron separados por electroforesis en gel de agarosa al 3,5\% (p/v) y teñidos con GelRed (Biotium, Inc. EUA). El gel fue visualizado en un transluminador bajo luz UV utilizando un marcador de peso molecular de 100 pb (Invitrogen company, USA). El cálculo de frecuencias genotípicas, alélicas y el equilibrio de Hardy Weinberg se realizó a través del software online ${ }^{1}$. El test de chi cuadrado fue determinado a través del software SPSS Statistics para Windows, versión 16,0 (SPSS Inc. Chicago. USA).

\section{RESULTADOS Y DISCUSIÓN}

Para detectar la mutación G1 se amplificó un fragmento de 462 pb, la digestión con la enzima HhaI generó los siguientes patrones: para el genotipo GG 254 pb, 156 pb y $52 \mathrm{pb}$, para el genotipo GA se generaron cuatro patrones: 410 pb, 254 pb, 156 pb y 52 pb (figura 1).

Para detectar la mutación G8 se amplificó un producto de PCR de $139 \mathrm{pb}$, la digestión con la enzima DdeI generó dos fragmentos de 108 pb y 31 pb, que corresponden a al genotipo CC (Figura 2). El número de alelos observados en los dos loci investigados, fueron similares a los reportes previos realizados por Hanrahan y col (2004).

www.husdyr.kvl.dk 
Cuadro 1. Secuencia de partidores utilizados para PCR-FLRP (Hanrahan y col 2004).

Primers used for PCR-FRLP (Hanrahan et al 2004).

\begin{tabular}{|c|c|c|c|}
\hline Gen & Mutación & Secuencia partidor $5^{\prime} \rightarrow 3^{\prime}$ & Cambio nucleotídico \\
\hline \multirow[t]{2}{*}{$\begin{array}{c}\text { GDF9 } \\
(\mathrm{AF} 078545)\end{array}$} & G1 & $\begin{array}{l}\text { F-GAAGACTGGTATGGGGAAATG- } \\
\text { R-CCAATCTGCTCCTACACACCT- }\end{array}$ & $\mathrm{G} \rightarrow \mathrm{A}$ \\
\hline & G8 & $\begin{array}{l}\text { F-CTTTAGTCAGCTGAAGTGGGACAAC } \\
\text { R-ATGGATGATGTTCTGCACCATGGTGTGAACCTGA }\end{array}$ & $\mathrm{C} \rightarrow \mathrm{T}$ \\
\hline
\end{tabular}

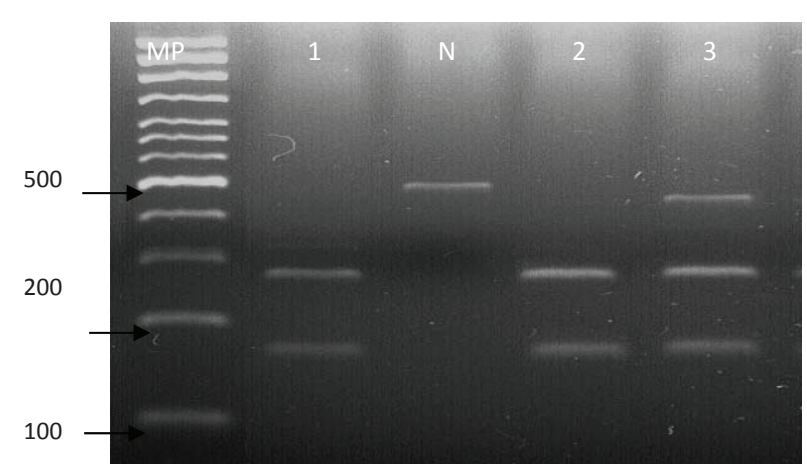

Figura 1. PCR-RFLP de la mutación G1 del gen GDF9 digerido con la enzima HhaI. Líneas 1 y 2: genotipo GG. Línea 3: genotipo GA. MP: marcador de peso molecular. N: control negativo.

PCF-RFLP products of GDF G1 by restriction enzyme Hhal. Lanes 1 and 2: genotype GG. Lane 3: genotype GA. MP: molecular weight marker. N: negative control.

La mutación G1 (figura 1) mostró ser polimórfica en la población de ovinos criollos Araucanos, con una frecuencia de 0,56 para el genotipo GG y de 0,44 para el genotipo GA. El cálculo de la frecuencia alélica fue de 0,78 para el alelo $\mathrm{G}$ y de 0,22 para el alelo A (cuadro 2). No se detectó para la mutación G1 la presencia de individuos homocigotos portadores del genotipo AA.

Estos resultados coinciden con reportes previos realizados en ovinos asiáticos, los cuáles promediaron frecuencias genotípicas de 0,66 para los individuos GG; 0,32 para los individuos GA y 0,02 para los individuos AA (cuadro 3). En algunos casos no fue posible detectar la presencia de individuos mutantes (Ghaderi y col 2010), lo cual coincide para el locus G8 en este estudio. No existen antecedentes filogenéticos que corroboren el parentesco de ovinos asiáticos y el criollo Araucano, sin embargo, se sabe que la población ovina de Chile tiene un origen ibérico, con una fuerte influencia de ovinos de origen británico introducidos al país a partir del siglo XIX (Correa 1938).

El test de chi cuadrado, indicó que la población no se encuentra en equilibrio de Hardy-Weinberg para esta mutación $\left(\chi^{2}=5,66\right)$. Esto se debe probablemente a que

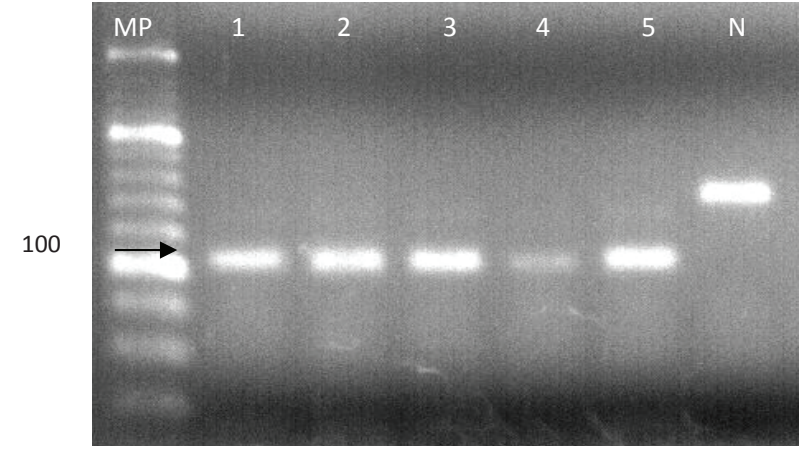

Figura 2. PCR-RFLP de la mutación G8 del gen GDF9 digerido con la enzima DdeI. Todos los productos (líneas 1 a 5) corresponden al genotipo CC. MP: marcador de peso molecular. N: control negativo.

PCR-FRLP products of GDF9 G8 by restriction enzyme DdeI. All products (lanes 1 to 5) correspond to genotype CC. MP: molecular weight marker. N: negative control.

existe una constante rotación de carneros con rebaños asociados y a una intensa selección de hembras en base a sus índices reproductivos. Esto provoca el descarte de individuos menos prolíficos o infértiles dentro de la población, lo que podría explicar la ausencia del genotipo AA para la mutación G1. GDF9 es un gen candidato importante debido a que está involucrado en el control de la tasa de ovulación y el consiguiente aumento del tamaño de la camada en el ganado ovino de diferentes razas (Silva y col 2010, Mahmoud y Nawito 2012). Existen estudios que indican la influencia del genotipo heterocigoto en el incremento del número de crías por parto (Hanrahan y col 2004, Barzegari y col 2010). También se ha señalado que las ovejas que son homocigotas para esta mutación son infértiles debido a que desarrollan una atresia ovárica (Hanrahan y col 2004). El polimorfismo G1 incrementaría el tamaño de la camada entre 1,3 y 1,7 crías más por parto (Javanmard y col 2011, Moradband y col 2011). Barzegari y col (2010), no encontraron una asociación entre los individuos mutados y esterilidad, aunque los animales que fueron estériles poseían ambas mutaciones tanto la variante G1 del gen GDF9 como la variante $F e c B^{G}$ del gen BMP15. 
PAZY COL

Cuadro 2. Frecuencia genotípica y alélica del polimorfismo G1 y G8.

Genotipic and allelic frequencies for G1 and G8 polymorphisms.

\begin{tabular}{cccccc}
\hline Polimorfismo & \multicolumn{2}{c}{ Frecuencia genotípica } & \multicolumn{2}{c}{ Frecuencia alélica } \\
\hline \multirow{2}{*}{ G8 } & CC & CT & TT & C & T \\
& 1 & 0 & 0 & 1 & 0 \\
G1 & GG & GA & AA & G & A \\
\hline
\end{tabular}

Cuadro 3. Frecuencias genotípicas y alélicas de G1 y G8 en diferentes razas.

Genotype and allelic frequencies of G1 and G8 in different breeds sheeps.

\begin{tabular}{|c|c|c|c|c|c|c|c|c|c|c|}
\hline \multirow{3}{*}{ Raza } & \multicolumn{3}{|c|}{ Frecuencia genotípica } & \multicolumn{3}{|c|}{ Frecuencia genotípica } & \multicolumn{2}{|c|}{ Frecuencia alélica } & \multicolumn{2}{|c|}{ Frecuencia alélica } \\
\hline & \multicolumn{3}{|c|}{ G1 } & \multicolumn{3}{|c|}{ G8 } & \multicolumn{2}{|c|}{ G1 } & \multicolumn{2}{|c|}{ G8 } \\
\hline & GG & GA & AA & $\mathrm{CC}$ & $\mathrm{CT}$ & TT & G & A & C & $\mathrm{T}$ \\
\hline Afshari ${ }^{1}$ & 0,52 & 0,48 & 0 & \multicolumn{3}{|c|}{ No hay datos } & 0,76 & 0,24 & \multicolumn{2}{|c|}{ No hay datos } \\
\hline Baluchi $^{1}$ & 0,61 & 0,33 & 0,06 & \multicolumn{3}{|c|}{ No hay datos } & 0,78 & 0,22 & \multicolumn{2}{|c|}{ No hay datos } \\
\hline Makui $^{1}$ & 0,57 & 0,40 & 0,03 & \multicolumn{3}{|c|}{ No hay datos } & 0,77 & 0,23 & \multicolumn{2}{|c|}{ No hay datos } \\
\hline Merhaban $^{1}$ & 0,64 & 0,36 & 0 & \multicolumn{3}{|c|}{ No hay datos } & 0,82 & 0,18 & \multicolumn{2}{|c|}{ No hay datos } \\
\hline Kordi $^{2}$ & 0,81 & 0,19 & 0 & 1 & 0 & 0 & 0,91 & 0,09 & 1 & 0 \\
\hline Arabic $^{2}$ & 0,84 & 0,16 & 0 & 1 & 0 & 0 & 0,92 & 0,08 & 1 & 0 \\
\hline Iranian Baluchi ${ }^{3}$ & 0,72 & 0,20 & 0,08 & \multicolumn{3}{|c|}{ No hay datos } & 0,82 & 0,18 & \multicolumn{2}{|c|}{ No hay datos } \\
\hline Chios $^{4}$ & 0,61 & 0,30 & 0,09 & 0,65 & 0,11 & 0,24 & 0,76 & 0,24 & 0,70 & 0,30 \\
\hline Karagouniki $^{4}$ & 0,95 & 0,05 & 0 & 0,76 & 0,24 & 0 & 0,97 & 0,03 & 0,88 & 0,12 \\
\hline
\end{tabular}

${ }^{1}$ Javanmard y col 2011; ${ }^{2}$ Ghaderi y col 2010; ${ }^{3}$ Moradband y col 2011; ${ }^{4}$ Liandris y col 2012.

El locus G8 fue monomórfico en la población estudiada (figura 2), encontrándose en la población evaluada solo el genotipo CC. Estudios realizados en razas prolíficas asiáticas tampoco mostraron la presencia de esta mutación, pero se ha detectado en razas europeas como Belclare y Cambridge (Hanrahan y col 2004, Ghaffari y col 2009). La mutación G8 también llamada $F e c G^{H}$ (high fertility) causa incrementos en la tasa ovulatoria en ovejas heterocigotas, mientras que ovejas homocigotas son estériles (Vacca y col 2010).

Los resultados obtenidos en este estudio permitieron solo la identificación del polimorfismo G1 del gen GDF9 en la población de ovinos criollos Araucanos. Este es el primer reporte de este polimorfismo en ovinos en Chile, investigaciones posteriores están orientadas a establecer estudios de asociación y determinar el impacto en el mejoramiento reproductivo de los rebaños al utilizarlo en la identificación temprana de individuos prolíficos.

\section{REFERENCIAS}

Barzegari A, S Atashpaz, K Ghabili, Z Nemati, M Rustaei, R Azarbaijani. 2010. Polymorphisms in GDF9 and BMP15 associated with fertility and ovulation rate in Moghani and Ghezel sheep in Iran. Reprod Dom Anim 45, 666-669.

Bravo S, N Sepúlveda. 2010. Índices zoometricos en ovejas criollas Araucanas. Int J Morphol 28, 489-495.

Chu M, Z Liu, C Jiao, Y He, L Fang, S Ye, G Chen, J Wang. 2007. Mutations in BMPR-IB and BMP-15 genes are associated with litter size in Small Tailed Han sheep (Ovis aries). J Anim Sci 85, 598-603.

Chu M, J Yang, T Feng, G. Cao, L Fang, R Di, D Huang, Q Tang, Y Ma, K Li, N Li. 2011. GDF9 as a candidate gene for prolificacy of Small Tail Han sheep. Mol Biol Rep 38, 5199-5204

Correa VL. 1938. La ganadería. En: Correa VL (ed). La agricultura Chilena. Tomo II. Imprenta Nascimiento, Santiago, Chile, Pp 195-198.

Davis G. 2004. Fecundity genes in sheep. Anim Reprod Sci 82, 
247-253.

Dekkers J. 2004. Commercial application of marker and geneassisted selection in livestock: strategies and lessons. $J$ Anim Sci 82, 313-328.

Elvin J, A Clark, P Wang, N Wolfman, M Matzuk. 1999. Paracrine actions of Growth Differentiation Factor-9 in the mammalian ovary. Mol Endocrinol 13, 1035-1048.

Flórez J. 1992. Fármacos analgésicos opiáceos. En: Flórez J (ed). Farmacología Humana. $2^{\mathrm{a}}$ ed. Masson-Salvat, Barcelona, España, Pp 25-28.

Ghaderi A, MT Beigi Nasari, KH Mirzadeh, J Fayazi, AS Sadr. 2010. Identification of the GDF9 mutation in two sheep breeds by using polymerase chain reaction-restriction fragment length polymorphism (PCR-RFLP) technique. Afr J Biotechnol 9, 8020-8022.

Ghaffari M, A Nejati-Javaremi, G Rahimi-Mianji. 2009. Lack of polymorphism in the oocyte derived growth factor (GDF9) gene in the Shal breed of sheep. S Afr J Anim Sci 4, 355-360.

Hanrahan J, S Gregan, P Mulsant, M Mullen, G Davis, R Powell, S Galloway. 2004. Mutations in the genes for oocyte-derived growth factors GDF9 and BMP15 are associated with both increased ovulation rate and sterility in Cambridge and Belclare sheep (Ovis aries). Biol Reprod 70, 900-909.

Javanmard A, N Azadzadeh, A Esmailizadeh. 2011. Mutations in bone morphogenetic protein 15 and Growth Differentiation Factor 9 genes are associated with increased litter size in fat-tailed sheep breeds. Vet Res Com 35, 157-167.

Knight P, C Glister. 2006. Focus on TGF- $\beta$ Signaling: TGF- $\beta$ superfamily members and ovarian follicle development. Reproduction 132, 191-206.

Liandris E, A Kominakis, M Andreadou, K Kapeoldassi, S Chadio, Th Tsiligianni, M Gazouli, I Ikonomopoulos. 2012.
Associations between single nucleotide polymorphisms of GDF9 and BMP15 genes and litter size in two dairy sheep breeds of Greece. Small Rumin Res 107, 16-21.

Mahmoud K, M Nawito. 2012. Molecular markers for fertility in farm animals. Iran J Appl Anim Sci 2, 203-222.

Moradband F, G Rahimi, M Gholizadeh. 2011. Association of polymorphisms in fecundity genes of GDF9, BMP15 and BMP15-1B with litter size in Iranian Baluchi sheep. Asian-Aust J Anim Sci, 24, 1179-1183.

Otsuka F, K Mctavish, S Shimasaki. 2011. Integral role of GDF-9 y BMP-15 in ovarian function. Mol Reprod Dev 78, 9-21.

Sepúlveda N. 1999. Características productivas de los rebaños ovinos de ganaderos indígenas Mapuches en la IX Región de Chile. El Arca 3, 47-52.

Sepúlveda N, R Neculmán, M Herrera, E Rodero. 2001. Parámetros de fertilidad natural en ovejas de la agrupación araucana. XXVI Jornadas Científicas y V Internacionales de la Sociedad Española de Ovinotecnia y Caprinotecnia, Pp 962-966.

Silva BDM, EA Castro, CJH Souza, SR Paiva, R Sartori, MM Franco, HC Azevedo, TASN Silva, AMC Vieira, JP Neves, EO Melo. 2010. A new polimorphism in the Growth and Differentiation Factor 9 (GDF9) gene is associated with increased ovulation rate and prolificacy in homozygous sheep. Anim Genet 42, 89-92

Vacca G, A Dhaouadi, M Rekik, V Carcangiu, M Pazzola, M Dettori. 2010. Prolificacy genotypes genotipes at BMPR1B, BMP15 and GDF9 genes in North African sheep breeds. Small Rumin Res 88, 67-71.

Varani S, J Elvin, C Yan, J DeMayo, F DeMayo, H Horton, M Byrne, M Matzuk. 2002. Knockout of pentraxin 3, a downstream target of Growth Differentiation Factor-9, causes female subfertility. Mol Endocrinol 16, 1154-1167. 
